\title{
A general method for creating realistic reduced compartmental models from electrophysiological traces Eric B Hendrickson* ${ }^{* 1,2}$, Jeremy R Edgerton ${ }^{1}$ and Dieter Jaeger ${ }^{1}$
} Address: ${ }^{1}$ Department of Biology, Emory University, Atlanta, GA 30322, USA and 2Department of Biomedical Engineering, Georgia Institute of
Technology, Atlanta, GA, 30332, USA

Email: Eric B Hendrickson* - eric.hendrickson@bme.gatech.edu

* Corresponding author

from Seventeenth Annual Computational Neuroscience Meeting: CNS*2008 Portland, OR, USA. 19-24 July 2008

Published: II July 2008

BMC Neuroscience 2008, 9(Suppl I):P83 doi:I0.1 186/I47I-2202-9-SI-P83

This abstract is available from: http://www.biomedcentral.com/I47I-2202/9/SI/P83

(c) 2008 Hendrickson et al; licensee BioMed Central Ltd.

Reduced compartmental models, i.e. those incorporating Hodgkin-Huxley ion channels and few dendritic compartments, are frequently used in computational neuroscience both individually and as parts of networks. These models are typically hand-tuned individually and different criteria for model performance have been used by different investigators. Here, we present a general method which uses a particle swarm evolutionary algorithm to constrain the morphological, passive and active parameters of a reduced model to fit the fI curve, spontaneous spike shape, and response to negative current injection of a specific slice recording. The free passive and morphological parameters were axial resistance, membrane resistance and capacitance, soma diameter, and proximal and distal dendritic diameter and length. The free active parameters were the somatic and dendritic conductance densities of $\mathrm{NaF}, \mathrm{NaP}, \mathrm{Kv} 2, \mathrm{Kv} 3, \mathrm{Kv} 4, \mathrm{KCNQ}, \mathrm{SK}, \mathrm{CaHVA}$ and HCN. Using this method, we created populations of six-compartment models that specifically matched one of three rat globus pallidus (GP) neuron recordings (Figures 1,2). A separate population of models was created to match the recordings of each of the three target neurons, and members of each population presented specific matches for the targeted recording. Thus, the population of models that were created to match the recordings of a particular neuron matched those recordings better than populations of models created to match the recordings of other neurons. Interestingly, we found that most of the average conductance densities were not significantly different between the populations of models created to match the recordings of different neurons. Additionally, we calculated the average

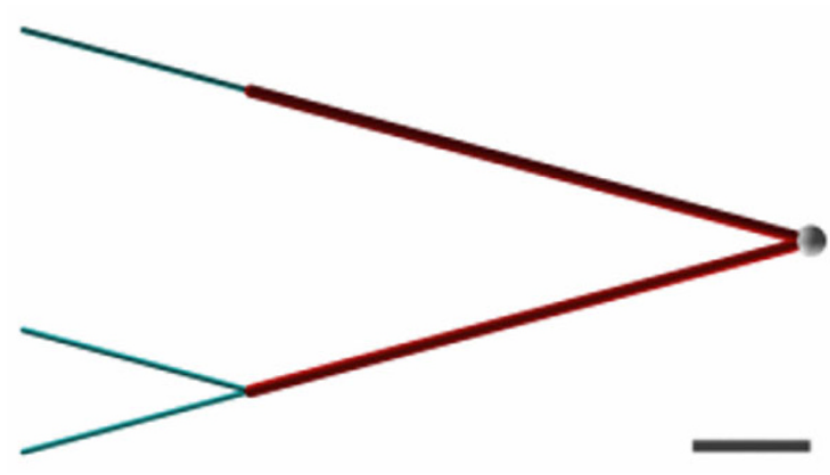

Figure I

Sample morphology of a six-compartment model.

parameter distance between populations of models with a normalized Euclidean distance measure (See equation in figure 3). Using this distance measure, the population of models created to match the recordings of a particular neuron was no closer to itself than it was to populations of models created to match the recordings of other neurons. This leads to the conclusion that the fitness spaces were well separated between matches for different neurons despite overlapping conductance parameter spaces. Overall, we find our method suitable for creating realistic reduced models of individual recordings for use in heterogeneous network simulations or hybrid experiments using dynamic clamp. 

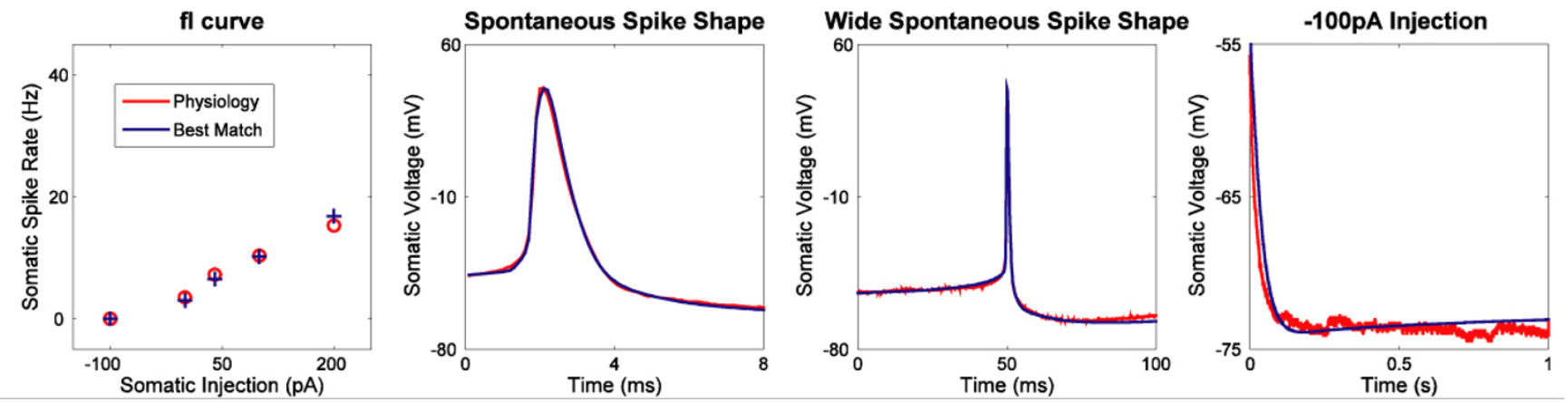

Figure 2

Output of the model that best matched the electrophysiological recordings of one test neuron.

$d i s t_{\text {normalizedEuclidean }}=\frac{\sum_{i=1}^{l} \sum_{j=1}^{m} \sqrt{\sum_{k=1}^{n}\left(\operatorname{swarm}_{1}(i, k)-\operatorname{swarm}_{2}(j, k)\right)^{2}}}{l * m}$

\section{Figure 3}

Normalized Euclidean distance measure. The distance between every model in swarm 1 (I models) and swarm $_{2}(\mathrm{~m}$ models) is summed, then divided by the total number of distances calculated $\left(I^{*} \mathrm{~m}\right)$. 